\title{
The Upgraded Microstrip Silicon Sensor Characterisation Facility of the University of Sheffield
}

\author{
E. Kourlitis*, K. Lohwasser, R. French and S. Edwards \\ The University of Sheffield, UK \\ E-mail: evangelos.kourlitis@cern.ch, kristin.lohwasser@cern.ch, \\ r.s.french@sheffield.ac.uk, s.o.edwardsesheffield.ac.uk
}

\begin{abstract}
A major component of the ATLAS Phase-II upgrade is the Inner Tracker, an all-silicon detector featuring novel microstrip sensors. Motivated by the current research efforts in silicon sensor technology, a characterisation facility has been recently upgraded at the University of Sheffield. A description of the facility along with initial benchmark measurements of sensors before and after their irradiation are presented. The results were found to be in agreement with similar measurements conducted by other facilities in the UK and support the successful commissioning of the facility.
\end{abstract}

Topical Workshop on Electronics for Particle Physics (TWEPP2018)

17-21 September 2018

Antwerp, Belgium

${ }^{*}$ Speaker. 


\section{Introduction}

Microstrip (strip) silicon sensors are used in a variety of fields, from physical research to medicine. In many cases, as high-energy physics experiments or space-based applications, the device radiation tolerance is crucial and performance should be adequate in harsh environments. Additionally, replacement after installation is often unrealistic, thus only extensively tested for its lifetime technology can be used on these demanding applications. Motivated by the upgrade of the ATLAS detector at CERN, in accordance to the HL-LHC upgrade of the Large Hadron Collider [1], a strip sensor characterisation facility has been re-commissioned, after improvements, at the University of Sheffield (UoS).

The HL-LHC is designed to deliver an order of magnitude higher instantaneous luminosity to increase the chance of observing rare physics phenomena. This comes at a price of higher detector occupancy and thus higher radiation levels during operation. In order that the ATLAS detector maintains high performance in these new extreme standards, an upgrade on the tracking system, among others (Phase-II upgrade), is essential. The current Inner Detector is going to be replace by the all-silicon Inner Tracker (ITk). The ITk consist of a pixel detector, at small radius close to the beam line, and a large-area strip tracking detector surrounding it. The latter expands in a four-layer barrel section and one end-cap on each side with six disks each.

Radiation simulation studies have shown that integrated fluence up to $10^{15} 1 \mathrm{MeV} \mathrm{n}_{e q} / \mathrm{cm}^{2}$ is expected at the innermost layer of the barrel section, located $405 \mathrm{~mm}$ away from the beam line, after a decade of operation [2]. As the strip sensors are required to sustain high tracking capabilities over the whole duration of the HL-LHC program, evaluating the deterioration of different technologies and design architectures is essential for the final choice.

The characterisation facility of the UoS is comparing key parameters of the sensors' operational performance before and after test irradiations to assess the significance of this damage. It is located at the clean-room of the Hicks Building of the UoS and provide both individual and collaborative measurements with similar facilities in the UK, e.g. Liverpool and Birmingham [3].

This document provides an overview of the facility (Section 3) along with commissioning measurements (Section 4), assessing its operation. A brief introduction on the radiation damage of a silicon sensor (Section 2) is given, motivating the particular measurements conducted, while a conclusion (Section 5) completes the document.

\section{Radiation Damage on Silicon Sensors}

High-energy particles that transverse a silicon sensor continuously damage it as, essentially, energy is also deposited in the material. It is useful to separate this damage into two categories as different macroscopic effects are caused depending on the sensor material affected: the bulk or the surface. The following paragraphs present few aspects of the radiation damage physics to motivate the measurements of the leakage current, charge collection efficiency and the cluster size in the evaluation of the performance of an irradiated strip sensor.

\section{Bulk damage}

Particles that cross the bulk material of a sensor can interact with the atoms of the crystal 
lattice, ionizing the atoms to induce a measured signal or displacing them to create interstitial and/or vacancy defects. The latter eventually alter the material's elementary properties and degrades its performance.

- Leakage current increases, as the defects act as electron-hole pair generation centres. This increase can be seen as excess heat that should be dissipated by cooling the sensors to keep this current, and thus the noise, low.

- Charge Collection Efficiency (CCE) decreases, due to defects acting as charge traps. The trapping rate is proportional to the number of defects and thus to the integrated fluence. At very high fluences $\left(\geq 10^{15} 1 \mathrm{MeV} \mathrm{n} \mathrm{n}_{e q} / \mathrm{cm}^{2}\right)$ this becomes the main limiting factor of silicon usage as charge is trapped before it arrives at the collection electrodes [4].

\section{Surface damage}

Surface damage describes the defects on the $\mathrm{SiO}_{2}$ insulating surface material. This is induced by ionisation, in contrast to the bulk damage, and it has more permanent nature. Electrons and holes have very different mobilities in the $\mathrm{SiO}_{2}$, thus after the ionisation are quickly separated and holes are accumulated to the $\mathrm{Si}-\mathrm{SiO}_{2}$ interface. Consequently, electrons are attracted from the bulk side towards the interface and accumulate there too. This configuration results in a decreased inter-strip resistance and increased cross-talk. Effectively the sensor's spatial resolution is deteriorated by measuring wider clusters, i.e. larger number of strip collects the charge induced.

\section{Characterisation Facility}

Two key elements of the characterisation of a sensor are its signal readout and testing environment. As the measurement is heavily dependent on both these factors, the design of the facility at the UoS has been driven to closely resemble the operational conditions on HL-LHC.

The sensors' signal is acquired using the ALiBaVa system [5]. The system is composed of three units, the daughterboard, a custom build triggering sub-system and the motherboard. The core of the system is the Beetle ASIC chip that readouts the strip sensor providing an analogue output that is sampled with the frequency of the chip clock (40 MHz). Two Beetle chips, sensor mountings and power supplies-low-voltage for the chips and high-voltage for the sensor-are all incorporated on the daughterboard shown in Figure 1 (left). In order to excite the sensor and measure the signal, a radioactive source emitting $\beta$-radiation $\left({ }^{90} \mathrm{Sr}\right)$ is used. It is placed right above the sensor so the particles are crossing it with the minimum solid angle, thus higher flux, as possible. Underneath the daughterboard, a custom-made system is triggering the ALiBaVa readout when the charged particles are crossing it. It is composed of two plastic scintillators mounted on photomultiplier and acting in coincidence to reduce the random environmental background. The whole setup is schematically shown in Figure 1 (middle). Finally, the purpose of the motherboard is to process the analogue data provided by the chip in accordance with the trigger signal. The latter allows the motherboard to read a particular position in the pipeline. Only one sample is needed for each event while the Landau pulse shape is reconstructed by acquiring enough events. The peak of the pulse is proportional to the collected charge by the sensor. The motherboard's FPGA controls the system and is interfaced with the computer. 
Exploiting the separability of the above three components, everything except of the motherboard (Figure 1 (middle)) is placed in a controlled environment. To resemble the operational conditions, measurements can be conducted at temperatures down to about $-30^{\circ} \mathrm{C}$ and humidity down to $10 \% \mathrm{RH}$. The environmental conditions are controlled by a fridge with dry nitrogen supply and monitored by a probe placed next to the under-test sensors. The whole facility described is depicted in Figure 1 (right), where the high and low voltage supplies can be also seen.
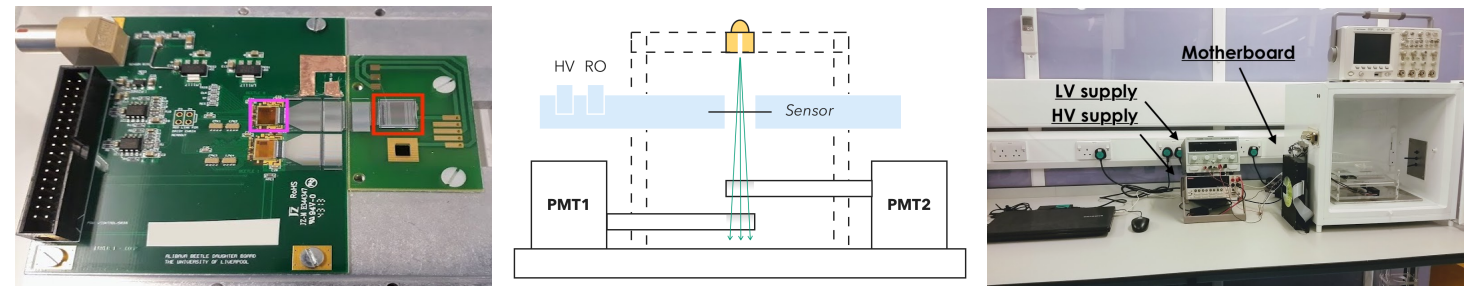

Figure 1: (left) The daughterboard with the Beetle chip (pink) and the sensor mounted on (red). (middle) The source, daughterboard and triggering setup. (right) The UoS strip sensor characterisation facility.

\section{Commissioning Measurements}

In order assess the operational performance of the facility, commissioning measurements have been taken comparing the leakage current, CCE and cluster size of an unirradiated sensor with two irradiated ones. The irradiation had been performed at the Birmingham MC40 irradiation facility [3] in 2014 with the purpose of defining benchmark irradiated sensors to compare among the characterisation facilities of the UK.

In particular, $\mathrm{n}^{+}$-in-p miniature sensors $(1 \mathrm{~cm} \times 1 \mathrm{~cm})$ of the ATLAS12 design [2], which have been fabricated to test the performance standards needed for the strip modules of the ITk are used. Two sensors were irradiated at fluence of $10^{15} 1 \mathrm{MeV} \mathrm{n}$ eq $/ \mathrm{cm}^{2}$ using a $24 \mathrm{MeV}$ proton beam of 1 $\mu \mathrm{A}$ current. The methodology of irradiation that was followed is referred to as scanning irradiation; the samples are placed in front of the incident beam and are scanned horisontally using a XY-axis robotic system. This mode allows beam non-uniformities to be eliminated. The two sensors were irradiated at different scanning speeds $(8 \mathrm{~mm} / \mathrm{s}$ and $1 \mathrm{~mm} / \mathrm{s})$ in order for potential thermal annealing effects during irradiation to be apparent on the measurements. The sensors have been subsequently stored and transferred in sub-zero temperatures to reduce any post-irradiation annealing effects to negligible levels.

Leakage measurements obtained from the irradiated sensors are presented in Figure 2 (left) and show an increase compared to the unirradiated sensor in which the maximum current measured at few nA. In Figure 2 (middle) the CCE curve as function of the bias voltage applied is shown, where a degradation of the performance of the irradiated sensors is apparent, compared to the unirradiated one. In particular, $65-80 \%$ lower CCE is measured on the full depletion regime (> $300 \mathrm{~V}$ ), depending on the thermal damage of the sensor during the irradiation. Finally, a cluster size analysis per event is performed; neighbor strips to the one with the highest signal, and Signal to Noise Ratio (SNR) greater than 3.5, are added to it if they pass the threshold of SNR greater than 1.8. In Figure 2 (right) can be seen a slight broadening of the cluster size distribution of the irradiated sensor compared to the unirradiated one. 

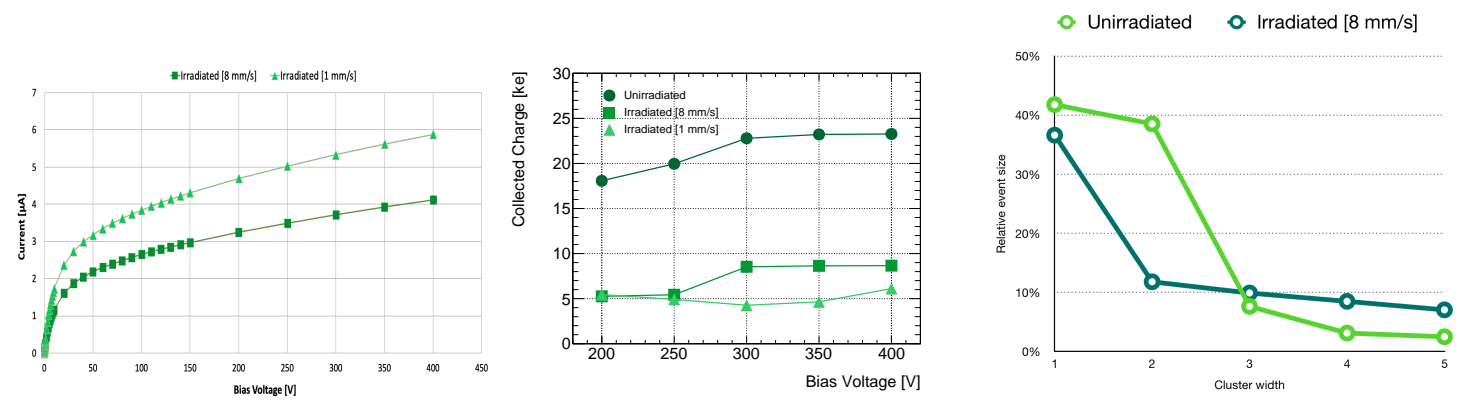

Figure 2: (left) The characteristic curves of the two irradiated sensors used for the leakage current measurement. (middle) The cluster size distribution of the unirradiated and the irradiated sensor with scanning speed of $8 \mathrm{~mm} / \mathrm{s}$ at $400 \mathrm{~V}$ bias. Each distribution is normalised to the total number of events per sensor measurement. (right) The CCE as function of the bias voltage applied for the unirradiated and the two irradiated ATLAS12 sensors.

Overall, it can be seen that the irradiated sensor that was scanned at the slower speed $(1 \mathrm{~mm} / \mathrm{s})$ has much lower measured performance, showing higher noise due to increased leakage current and lower CCE. Generally, all the measurements found in agreement with other facilities in the UK [3] and abroad [2].

\section{Conclusion}

A description of the recently upgraded silicon strip sensor characterisation facility at the UoS was presented. The facility is using an analogue readout chip to measure the sensor's signal while the tests are performed in a controlled environment resembling the operational one. Benchmark sensors were measured before and after their irradiation in order to assess the performance of the facility. Leakage current, CCE and cluster size measurements show reasonable agreement with equivalent ones conducted by other facilities at the same type and conditions of sensors, and thus support the successful commissioning of the facility.

\section{References}

[1] L. Rossi and O. BrÃijning, High Luminosity Large Hadron Collider : A description for the European Strategy Preparatory Group, CERN-ATS-2012-236.

[2] ATLAS Collaboration, Technical Design Report for the ATLAS Inner Tracker Strip Detector, CERN-LHCC-2017-005.

[3] P. Allport, et al., Recent results and experience with the Birmingham MC40 irradiation facility, JINST $12 \mathrm{C} 03075$

[4] F. Hartmann, Evolution of Silicon Sensor Technology in Particle Physics, Springer International Publishing, Cham, 2017

[5] R. Marco-Hernandez, A Portable Readout System for Microstrip Silicon Sensors (ALIBAVA), IEEE Tran. Nuclear Science, Vol. 56, No. 3, June 2009. 